\title{
Commentary on Lumbar Lordosis in Chronic Mechanical Back Pain
}

\author{
Manish Kundanmal Kothari ${ }^{1}$, Pramod Saini ${ }^{1}$, Agnivesh Tikoo ${ }^{2}$ \\ ${ }^{1}$ Department of Orthopaedics, P D Hinduja National Hospital \& MRC, Mumbai, India \\ ${ }^{2}$ Department of Orthopaedics, Wockhardt Hospitals (South Mumbai), Mumbai, India
}

Dear Editor,

We read the study by Ashraf et al. [1] titled "Correlation between Radiologic Sign of Lumbar Lordosis and Functional Status in Patients with Chronic Mechanical Low Back Pain" published in the October 2014 issue with great interest. However, we believe there were a few aspects that were not adequately addressed.

First and foremost, in the inclusion and exclusion criteria, the authors did not account for the presence or absence of disc height collapse, a factor known to directly affect lumbar lordosis (LL). It would have been useful to examine disc condition using magnetic resonance imaging and exclude patients with advanced disc degeneration (Pfirrmann grade IV and above). Also, paraspinal muscle spasm and hamstring tightness are known to result in temporary loss of LL. Thus, these factors should have been part of the exclusion criteria.

Secondly, although the title of the study indicates that it is about a correlation between radiological LL and functional status using the Persian version of the Oswestry disability index (ODI), the authors did not present any values for the ODI in their results or tables. They presented an average LL of $44.69 \pm 11.13$ degrees. They also presented 23 subjects with LL greater than 56.12 degrees and 20 subjects with LL less than 36.26 degrees. The values of ODI in these outlier patients groups should have been presented and statistically compared to those within the range.

Thirdly, this study has a skewed male to female ratio $(\mathrm{F}: \mathrm{M}=119: 31)$. There is an obvious selection bias here which the authors did not explain. Also, the ages of the subjects ranged from 15 to 85 years. The authors should have excluded subjects over 60 years old as this population is prone to osteoporosis, a known cause of back pain.

Fourthly, the authors mentioned multiple factors that can affect LL in their introduction but did not feel the need to study any of the factors to ensure a homogenous group of subjects.

We agree with the authors that it is irrational to compare their study with studies based on spinal deformity as they are completely different pathologies that cannot be compared.

Lastly and most importantly, Roussouly et al. [2] suggested that LL as a parameter should not be studied alone. They proposed a promising classification of LL based on the orientation of the sacral slope. Based on the biomechanics of these curve types, curves prone to various spine pathologies were described [3]. We believe that the incidence of chronic mechanical back pain in these

Received Dec 20, 2014; Accepted Dec 20, 2014

Corresponding author: Manish Kundanmal Kothari

Department of Orthopaedics, P D Hinduja National Hospital \& MRC, 1408, Hinduja Clinic, Veer Savarkar Marg, Mahim, Mumbai, 400016, India 
curve types and vice versa should be studied for better understanding of its pathophysiology.

\section{Conflict of Interest}

No potential conflict of interest relevant to this article was reported.

\section{References}

1. Ashraf A, Farahangiz S, Pakniat Jahromi B, Setayes- hpour N, Naseri M, Nasseri A. Correlation between radiologic sign of lumbar lordosis and functional status in patients with chronic mechanical low back pain. Asian Spine J 2014;8:565-70.

2. Roussouly P, Berthonnaud E, Dimnet J. Geometrical and mechanical analysis of lumbar lordosis in an asymptomatic population: proposed classification. Rev Chir Orthop Reparatrice Appar Mot 2003;89:632-9.

3. Roussouly P, Pinheiro-Franco JL. Biomechanical analysis of the spino-pelvic organization and adaptation in pathology. Eur Spine J 2011;20 Suppl 5:609-18. 